\title{
Investigation of the effect of Lactobacillus paracasei TD3 on cutaneous wound healing and induction of angiogenesis on male Wistar rats
}

\author{
Abolfazl Dehkohneh¹, Ahmad Jiriaie², Omid Ardalani33, Amin Mohsenzadeh4, Hoda Sabati5, Parvaneh \\ Jafari6,** \\ ${ }^{1}$ Department of Biotechnology, Faculty of Advanced Science and Technology, Tehran Medical Sciences, Islamic Azad University, \\ Tehran, Iran \\ ${ }^{2}$ Microbiology Department, Faculty of Science, Islamic Azad University, Arak Branch, Arak, Iran \\ 3Department of Microbial Biotechnology, School of Biology and Centre of Excellence in Phylogeny of Living Organisms, College of \\ Science, University of Tehran, Tehran, Iran \\ 4 Department of Microbiology, Faculty of Science, Ardabil Branch, Islamic Azad University, Ardabil, Iran \\ 5 Biotechnology and Biological Science Research Center, Faculty of Science, Shahid Chamran University of Ahvaz, Ahvaz, Iran \\ ${ }^{6}$ Department of Food Safety, Food Security Research Institute, Islamic Azad University, Arak Branch, Arak, Iran
}

\begin{abstract}
Skin plays an essential role in the prevention of pathogenic microorganisms entrance. It is also considered as the first line of immune defense in our body. The therapeutic effects of probiotics on wound healing are well reported over the past decade. Nevertheless, there are limited publications about the impact of probiotics on skin wound healing. Here we assessed the effect of Lactobacillus paracasei TD3 on cutaneous wound healing in male Wistar rats. During this research, thirty-six male Wistar rats grouped into control positive, control negative, and trial groups, then the skins of rats were incised to make a full-thickness wound. An ointment produced from probiotic L. paracasei TD3 was administrated to the trial group, and immunohistological factors of each host were evaluated and compared with control groups. The final results showed that although $L$. paracasei TD3 could proceed the wound closure earlier than other groups and could induce angiogenesis in trial group, it could not cause any significant changes in the levels of monocytes, lymphocyte, mast cells and polymorphonuclear leukocytes in the trial group compared to control groups. This probiotic bacterium may be effective besides other probiotic bacteria.
\end{abstract}

Keywords: Probiotics, Wound healing, Lactobacillus paracasei TD3, Skin, Probiotic ointment

\section{Introduction}

Skin plays an essential role in the prevention of pathogenic microorganisms' entrance. It is also considered as the first line of immune defense in our body $[1,2]$. The wound causes the skin to lose its integrity, thus activate a cascade of an event that led to wound healing. Wound repair process includes three overlapping phases, namely inflammation, granulation, and remodeling [3, 4]. On the other hand, recently, some authors describe the four-phase concept for wound healing, including hemostasis, inflammatory, proliferation, and remodeling, respectively $[5,6]$.

\section{*Corresponding author:}

Parvaneh Jafari, Ph.D

Department of Food Safety, Food Security Research Institute,

Islamic Azad University, Arak Branch, Arak, Iran

(C) The Author(s) 2021

Tel/Fax: +98 8634132451

Email: : p-jafari@iau-arak.ac.ir

https://orcid.org/oooo-0003-2771-9309

Received: April, 25, 2021

Accepted: June, 26, 2021 
There are several strategies to improve one or more phases to accelerate the wound healing process. Since chemical agents have adverse side effects, the tendency to use a novel alternative method is increased [5, 7, 8]. An appropriate agent should accelerate the wound repair process to prevent infection as well as angiogenesis induction. More recently, some authors evaluate the effect of different strains of probiotics as a therapeutic agent. According to the world health organization (WHO) definition, probiotics have beneficial effects on the host when they are consumed adequately. Probiotics are humanfriendly bacteria that have healthy effects on the human body, including reduction of serum cholesterol, immune-system improvement, anticancer properties, and prevention of body infection [9]. In this study, we investigated the effects of $L$. paracasei TD3, a native Iranian probiotics, on wound healing and induction of angiogenesis. Moreover, we evaluated the level of production of lymphocytes, mast cells, polymorphonuclear leukocyte, and monocytes, besides examination of the epithelialization, edema, inflammation and granulation.

\section{Method and Materials}

\subsection{Animals and feeding}

Thirty-six male Wistar rats weighing 150-180 g and 3-4 weeks old were purchased from Baqiat-Allah research center. They were divided into three groups $(\mathrm{n}=12)$ after 10 days acclimatization, negative control (NC), positive control (PC), and trial (probiotic). The tested groups were housed under the controlled condition of temperature $\left(22-25^{\circ} \mathrm{C}\right)$, light $(12 \mathrm{~h}$ light and $12 \mathrm{~h}$ dark), and humidity (20\%-30\%). In the whole experiment period, all the rats had free access to both food and water. The rats were fed by autoclaved food, which protein (23\%), salt (0.55\%), and lysine (1.15\%) were the most abundant ingredients on their food. After incision, they were killed at 3, 7, 14, and 21 days. Our study was carried out according to the approved protocol by the Institutional Animal Care and Use Committee (IACUC).

\subsection{Bacteria strain}

In this study, the lyophilized bacterium was purchased from Takgen Zist Company (Tehran, Iran). To prepare the ointment, $4.8 \mathrm{~g}$ lyophilized bacteria were mixed with $1000 \mathrm{~g}$ eucerin.

\subsection{Anesthesia and wounding process}

All the rats were anesthetized with an injection of a mixture of xylazine $(15 \mathrm{mg} / \mathrm{kg})$ and ketamine $(20 \mathrm{mg} / \mathrm{kg}$ ) into the peritoneum. The dorsal area was shaved and disinfected by alcohol (70\%) under anesthesia, and the skins of tested rats were incised to make a full-thickness wound approximately $1.5 \mathrm{~cm}^{2}$ by a surgical blade. After the wounding, the rats were housed in a separate sterilized cage. The wound treatments were carried out in tested groups once daily. For each wound, 19 of prepared ointment was applied to the probiotic group. For the control group, $1 \mathrm{~g}$ of probiotic free eucerin was used. After one day the wounding; NC without any treatment, PC was treated with eucerin only and trial (probiotic) was treated with eucerin plus L. paracasei TD3 strain (10 $12 \mathrm{CFU})$.

\subsection{Ulcer size assessment}

Assessment of the ulcer area and percentage of the ulcer healing was performed. Length and width measurements of the wound (mm2) were performed in days $1,3,7,14$, and 21 . The percentage of healing was calculated as below:

$$
\begin{aligned}
& \text { Percentage of healing }= \\
& \frac{\text { (Ulcer area on the first day) }-(\text { Ulcer area in the specific day) }}{\text { (Ulcer area in first day) }} \times 100
\end{aligned}
$$

\subsection{Histological examination}

Rats were sacrificed on days 1, 3, 7, 14 and 21, and tissues from the wound site was removed. The tissue samples were sectioned out and fixed overnight in 10\% neutral buffered formalin, then dehydrated gradually in ethanol and embedded in paraffin. Sections of $5 \mathrm{~mm}$ were obtained in an automated microtome. Ulcerated sections were stained with hematoxylin-eosin (H\&E) for histological evaluation during healing.

\subsection{Statistical analysis}

Statistical analysis was performed using the ANOVA test to determine significant differences between groups. Data were analyzed using Graph Pad software version 6. In entire experiment the P-value less than 0.05 means significant alteration.

\section{Results}

\subsection{Monocyte production}

Monocyte production was found to decrease significantly on day 14 in the trial group compared to control negative group ( $\mathrm{P}$ value $<0.05)$. This change was not significant between the trial group and the 
control positive group. In day 3 and 21 monocyte levels in trial group was higher than both control positive and control negative group, but this change was not statistically significant. On day 7 , monocyte production in the trial group was lower than two other groups, but this change was not statistically significant. Data are shown in Figure 1.

\subsection{Lymphocyte production}

Lymphocyte production in the trial group on day 3 was higher than control negative, and on days 7, 14 and 21, this factor was lower in the trial group in comparison with the control negative group. Although changes in the level of lymphocyte production were observed, these changes were not statistically significant. Data are shown in Figure 2.

\subsection{Mast cell production}

A gradual increase was observed in the level of Mast cell production in all of the experimental groups from day 3 to day 21. The highest mast cell production belonged to control positive group on day 21. On day 7 the level of mast cell production in the trial group was higher than two control groups, but differences between groups were not statistically significant in all stages of the experiment. Data are shown in Figure 3.

\subsection{Polymorphonuclear leukocyte production}

A gradual decrease was observed in the level of polymorphonuclear leukocyte production in all of the experimental groups from day 3 to day 21. The lowest polymorphonuclear leukocyte production was observed in the control negative group on day 21. On day 7 the level of polymorphonuclear leukocyte production in the trial group was higher than two control groups, but differences between groups were not statistically significant in all stages of the experiment. Data are shown in Figure 4.

\subsection{Granulation}

A gradual decrease was observed in the level of granulation in three experimental groups from day 3 to day 21. The lowest granulation was observed in the control negative group on day 21. On day 14 the level of granulation in the trial group was higher than two control groups, but differences between groups were not statistically significant in all stages of the experiment. Data are shown in Figure 5.

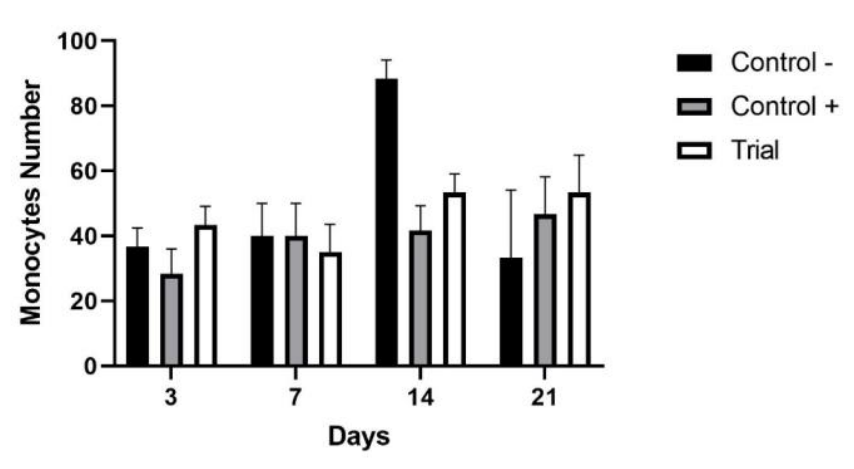

Figure 1. Comparison of monocyte production between three experimental groups. On day 14, the number of monocytes in control positive and the trial group was significantly lower than the control negative group.

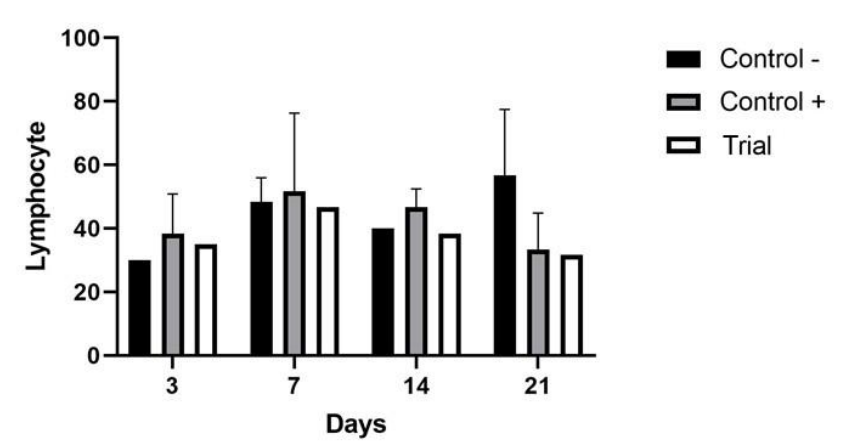

Figure 2. Comparison of lymphocyte production between three experimental groups. Although lymphocyte production in three groups was different, these differences were not statistically significant.

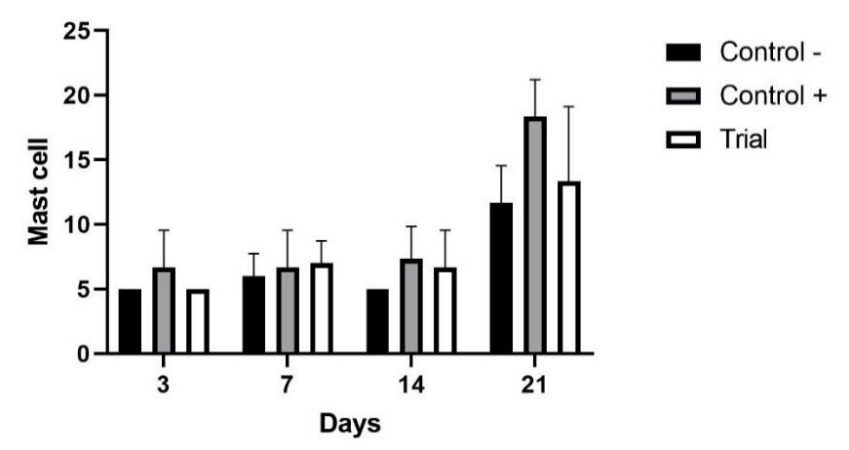

Figure 3. Comparison of mast cell production between three experimental groups. Although the Mast cell production increased gradually during the time in all of the experimental groups, there was no significant difference between all experimental groups. 


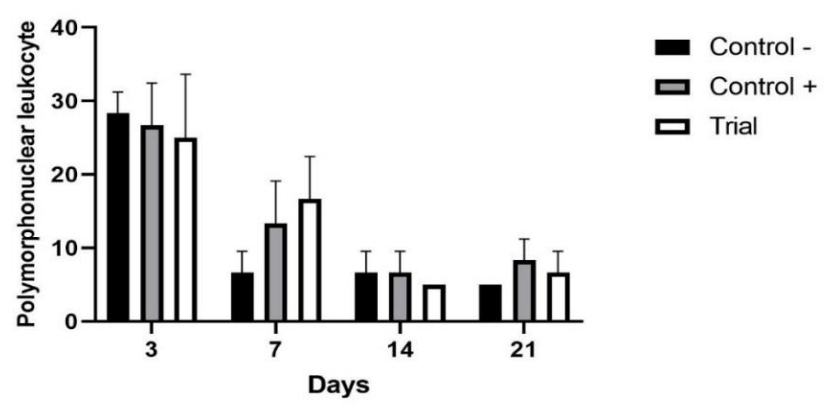

Figure 4. Comparison of polymorphonuclear (PMN) leukocyte production between three experimental groups. Although the PMN leukocyte production decreased gradually during the time in all of the experimental groups, there was no significant difference between all experimental groups.

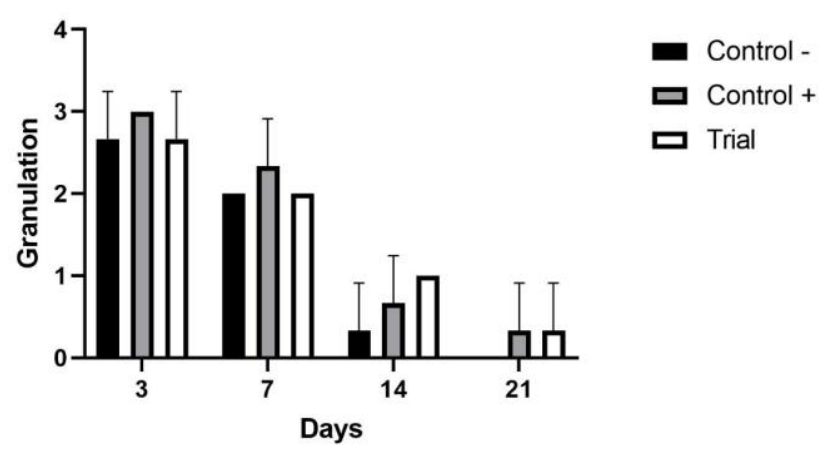

Figure 5. Comparison of granulation between three experimental groups. Although the granulation decreased gradually during the time in all of the experimental groups, there was no significant difference between all experimental groups.

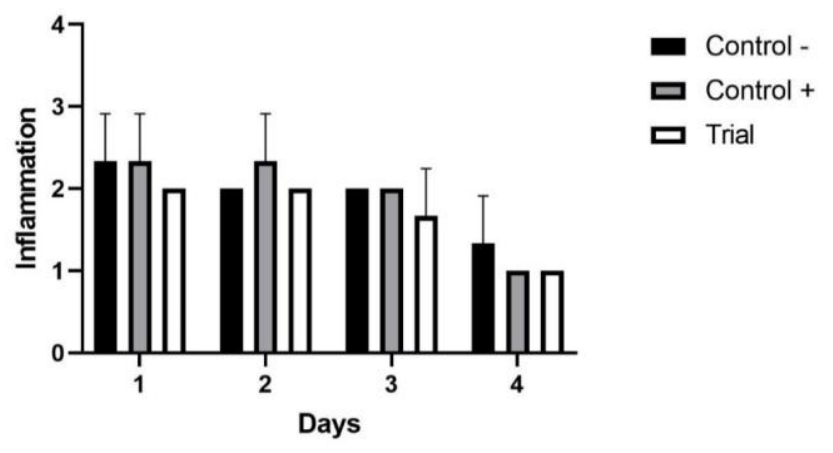

Figure 6. Comparison of inflammation between three experimental groups. Although the inflammation decreased gradually during the time in all of the experimental groups, there was no significant difference between all experimental groups.

\subsection{Inflammation}

A gradual decrease was observed in the level of inflammation in three experimental groups from day 3 to day 21. The lowest inflammation was observed in the trial group on day 21. Although during the experiment, thelevel of inflammation in the trial group was lower than the two other groups, differences between groups were not statistically significant in all stages of the experiment. Data are shown in Figure 6.

\subsection{Edema}

A gradual decrease was observed in the level of edema in three experimental groups from day 3 to day 21. The lowest edema was observed in the trial group on day 21. Although during the experiment, the level of edema was different between three experimental groups, these differences were not statistically significant in all stages of the experiment (Figure 7).

\subsection{Epithelialization}

A gradual increase was observed in the level of epithelialization in three experimental groups from day 3 to day 21. The highest epithelialization was observed in the control negative group on day 21. Although, during the experiment, the level of epithelialization was different between three experimental groups, these differences were not statistically significant in all stages of the experiment (Figure 8).

\subsection{Fibrosis}

A gradual increase was observed in the level of fibrosis in three experimental groups from day 3 to day 14. After that, a slight decrease was observed in all experimental groups on day 21. Although, during the experiment, the level of epithelialization was different between three experimental groups, these differences were not statistically significant in all stages of the experiment. Data are shown in Figure 9

\subsection{The progress of wound healing and angiogenesis}

The progress of wound healing among all experimental groups were compared. Based on macroscopic closure of the wounds, the wound healing in control groups were impaired, while the wounds were fully healed in probiotics group in 21 days after incision. Also, microscopic evaluation has revealed 
that $L$. paracasei could induce angiogenesis during wound healing (Supplementary figure 1, and 2).

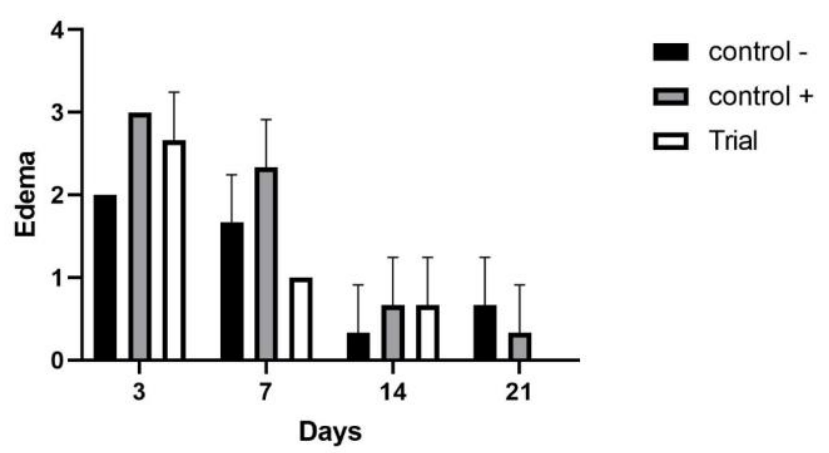

Figure 7. Comparison of edema between three experimental groups. Although the edema decreased gradually during the time in all of the experimental groups, there was no significant difference between all experimental groups.

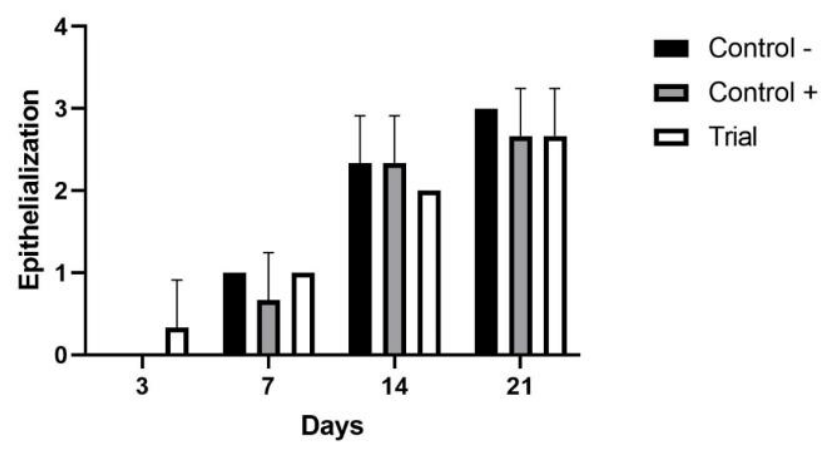

Figure 8. Comparison of epithelialization between three experimental groups. Although the epithelialization increased gradually during the time in all of the experimental groups, there was no significant difference between all experimental groups.

\section{Discussion}

Several approaches have been developed for wound healing acceleration [10-12]. These approaches might be involved in each of the three stages of wound healing, including inflammation, tissue formation, and tissue remodeling [13]. The therapeutic effects of probiotics on wound healing are well reported over the past decade [14-16]. Nevertheless, there are limited publications about the impact of probiotics on skin wound healing. The mechanisms of action of probiotics are still unclear. Moreover, it seems probiotic bacteria have more effects when they administered together. In the present study, the effect of $L$. paracasei TD3 on skin wound healing and immunological impacts of bacterium on its host was assessed. Our results showed that the administration of $L$. paracasei TD3 would not make any statistically significant change in the wound healing process and immunological factors of its host in comparison with control negative and control positive group while it could induce angiogenesis in trial group. There are limited studies on wound healing properties of $L$. paracasei TD3. Similar to our effort, Brandi et al. examined the effect of $L$. paracasei TD3 on wound healing. Their results on re-epithelialization did not show any significant change compared to control group, which was similar to our results [17]. Despite our work, the effect of other members of Lactobacillus on skin wounds showed an acceleration in the wound healing process. It has been reported that Lactobacillus brevis, and Lactobacillus plantarum might significantly accelerate wound healing and decrease inflammatory factors in rats after 21 days [18]. In another study, Khodaii et al. showed that Lactobacillus reuteri is capable of increasing reepithelialization in the rat model. They also reported that the probiotic group showed a lower inflammation rate compared with the control group [19]. Gudadappanavar et al. also showed that Lactobacillus acidophilus would accelerate wound healing by reducing epithelialization time in rat models [20].

Immune regulation functions of probiotics relate to the activation of natural killer (NK) cells, dendritic cells (DC), intraepithelial $\gamma \delta \mathrm{T}$ lymphocytes and macrophages, which are effector cells of innate immunity important for skin [21]. There are many reports about wound healing properties of probiotics, which mainly shows they might be considered as a new therapeutic agent in wound healing treatments [17-20]. Probiotics improve wound healing, while acting at the epidermis and dermis levels, where they function as signaling receptors against pathogens and activate the production of beta-defensins that increase the immunity of the skin [22]. Further studies should be considered for investigation of probiotic's safety, efficacy, and the mechanism of action in wound healing. Also potential side effects include allergic reactions to inactive ingredients, bacteremia, and antibiotic resistance transfer among pathogens must be reviewed which was not studied due to time 
constraints, materials and restrictions on access to sufficient number of rat models.

Todays, emerging scientific evidence has showed that probiotics may help wound healing. The present study investigated the effects of $L$. paracasei TD3, a native Iranian probiotic, on wound healing and induction of angiogenesis. Despite our results suggest induction of angiogenesis by the bacterium, there is a need for further studies on the probiotic bacterium and duration of its consumption. Our study about the effects of $L$. paracasei TD3 on wound healing and immunological factors in wistar rats did not show any statistically significant result, but it might be effective on other animal models. Further researches should be performed to gain a complete insight into the immunohistological and wound healing properties of L. paracasei TD3.

\section{Supplementary files}

Supplementary file 1.

\section{Author contributions}

$\mathrm{AD}$ : Designed and performed experiments, analyzed data and co-wrote the paper. AJ: Designed and performed experiments, analyzed data. OA, AM, HS: analyzed data and co-wrote the paper. PJ: Designed experiments, Supervised the research. All authors read and approved the final version of paper.

\section{Conflict of interests}

The authors declare that they have no conflicts of interest.

\section{Ethical declarations}

All procedures were performed in accordance with the guidelines of the Medical Ethics Committee of Islamic Azad University of Arak, Arak, Iran.

\section{Financial support}

None to be declared.

\section{References}

1. Cardoso CR, Favoreto S, Jr., Oliveira LL, Vancim JO, Barban GB, Ferraz DB, et al. Oleic acid modulation of the immune response in wound healing: a new approach for skin repair. Immunobiology. 2011; 216(3):409-15.

2. Bourke CD, Prendergast CT, Sanin DE, Oulton TE, Hall RJ, Mountford AP. Epidermal keratinocytes initiate wound healing and pro-inflammatory immune responses following percutaneous schistosome infection. Int J Parasitol. 2015; 45(4):215-24.
3. Janis JE, Kwon RK, Lalonde DH. A practical guide to wound healing. Plast Reconstr Surg. 2010; 125(6):230e-44e.

4. Velnar T, Bailey T, Smrkolj V. The wound healing process: an overview of the cellular and molecular mechanisms. J Int Med Res. 2009; 37(5):1528-42.

5. Guo S, Dipietro LA. Factors affecting wound healing. J Dent Res. 2010; 89(3):219-29.

6. Sinno H, Prakash S. Complements and the wound healing cascade: an updated review. Plast Surg Int. 2013; 2013:146764. 7. Weinstein-Oppenheimer CR, Aceituno AR, Brown DI, Acevedo C, Ceriani R, Fuentes MA, et al. The effect of an autologous cellular gel-matrix integrated implant system on wound healing. J Transl Med. 2010; 8:59.

8. Sasidharan S, Nilawatyi R, Xavier R, Latha LY, Amala R. Wound healing potential of Elaeis guineensis Jacq leaves in an infected albino rat model. Molecules. 2010; 15(5):3186-99.

9. Nagpal R, Kumar A, Kumar M, Behare PV, Jain S, Yadav H. Probiotics, their health benefits and applications for developing healthier foods: a review. FEMS Microbiol Lett. 2012; 334(1):1-15. 10. Lam EK, Yu L, Wong HP, Wu WK, Shin VY, Tai EK, et al. Probiotic Lactobacillus rhamnosus GG enhances gastric ulcer healing in rats. Eur J Pharmacol. 2007; 565(1-3):171-9.

11. Mimura T, Rizzello F, Helwig U, Poggioli G, Schreiber S, Talbot IC, et al. Once daily high dose probiotic therapy (VSL\#3) for maintaining remission in recurrent or refractory pouchitis. Gut. 2004; 53(1):108-14.

12. Sazawal S, Hiremath G, Dhingra U, Malik P, Deb S, Black RE. Efficacy of probiotics in prevention of acute diarrhoea: a metaanalysis of masked, randomised, placebo-controlled trials. Lancet Infect Dis. 2006; 6(6):374-82.

13. Singer AJ, Clark RA. Cutaneous wound healing. N Engl J Med. 1999; 341(10):738-46.

14. Choi HJ, Ahn JH, Park SH, Do KH, Kim J, Moon Y. Enhanced wound healing by recombinant Escherichia coli Nissle 1917 via human epidermal growth factor receptor in human intestinal epithelial cells: therapeutic implication using recombinant probiotics. Infect Immun. 2012; 80(3):1079-87.

15. Pinchuk IV, Bressollier P, Verneuil B, Fenet B, Sorokulova IB, Mégraud F, et al. In vitro anti-Helicobacter pylori activity of the probiotic strain Bacillus subtilis 3 is due to secretion of antibiotics. Antimicrob Agents Chemother. 2001; 45(11):3156-61.

16. Rolfe RD. The role of probiotic cultures in the control of gastrointestinal health. J Nutr. 2000; 130(2S Suppl):396s-402s.

17. Brandi J, Cheri S, Manfredi M, Di Carlo C, Vita Vanella V, Federici F, et al. Exploring the wound healing, anti-inflammatory, anti-pathogenic and proteomic effects of lactic acid bacteria on keratinocytes. Sci Rep. 2020; 10(1):11572.

18. Zahedi F, Heydari Nasrabadi M, Tajabadi Ebrahimi M, Aboutalebi H. Comparison of the effects of Lactobacillus brevis and Lactobacillus plantarum on cutaneous wound healing in rats.J Food Process Technol. 2012; 3(10):157.

19. Khodaii Z, Afrasiabi S, Hashemi SA, Ardeshirylajimi A, Natanzi MM. Accelerated wound healing process in rat by probiotic Lactobacillus reuteri derived ointment. J Basic Clin Physiol Pharmacol. 2019; 30(3).

20. Gudadappanavar AM, Hombal PR, Timashetti SS, Javali SB. Influence of Lactobacillus acidophilus and Lactobacillus plantarum on wound healing in male Wistar rats - an experimental study. Int J Appl Basic Med Res. 2017; 7(4):233-8. 


\section{Dehkohneh et al.}

21. Lukic J, Chen V, Strahinic I, Begovic J, Lev-Tov H, Davis SC, et al. Probiotics or pro-healers: the role of beneficial bacteria in tissue repair. Wound Repair Regen. 2017; 25(6):912-22.

22. Fijan S, Frauwallner A, Langerholc T, Krebs B, Ter Haar Née

Younes JA, Heschl A, et al. Efficacy of Using Probiotics with
Antagonistic Activity against Pathogens of Wound Infections: An Integrative Review of Literature. Biomed Res Int. 2019; 2019:7585486. 